\title{
Model-based Prediction of the Remaining Useful Life of the Machines
}

\author{
Pavle Boškoski ${ }^{1 *}$, Boštjan Dolenc ${ }^{1,2}$, Bojan Musizza ${ }^{1}$, Đani Juričić 1,3 \\ 1 Department of Systems and Control, Jožef Stefan Institute, Jamo va 39, 1000 Ljubljana, Slovenia;* pavle.boskoski@ijs.si \\ 2 Jo žef Stefan International Postgraduate School, Jamova 39, 1000 Ljubljana, Slovenia; \\ 3 University of Nova Gorica, Vipavska 13, 5000 Nova Gorica, Slovenia
}

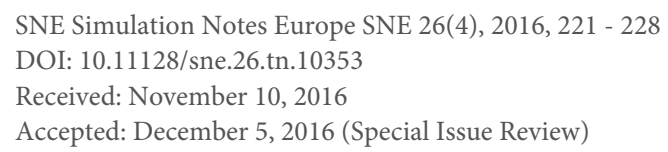

SNE Simulation Notes Europe SNE 26(4), 2016, 221 - 228

DOI: $10.11128 /$ sne.26.tn. 10353

Received: November 10, 2016

Accepted: December 5, 2016 (Special Issue Review)

Abstract. Accurate anticipation of the remaining useful life (RUL) of a machine is becoming mandatory for efficient exploitation of the asset and avoiding the unplanned downtimes. This should be achieved without extra investments in additional sensors and processing power. In this paper we present an approach to the RUL prediction of a shot blasting machine by using recordings from inexpensive vibrational sensors. The key idea consists in (i) employing generalised Jensen-Rényi divergence (JRD) as a measure of change in the vibrational pattern and (ii) associating JRD with the abrasive wear in rotor blades. It is essential to note that these two show monotonic relationship. Hereupon, a simple hidden Markov model with stochastic inputs and JRD as output is proposed. The hidden states of the model are updated on-line by means of Kalman filter. Prediction of the remaining useful life is done by executing Monte Carlo simulations on the updated model and evaluation of the first passage time of the JRD. The approach is successfully validated experimentally by running the machine up to failure, hence allowing for naturally evolving wear progression and breakdown.

\section{Introduction}

Stable and anticipative condition of process equipment, high availability and reliability, along with product quality are key factors that allow companies to stay competitive on the market. However, wear, material stress and environmental factors cause equipment to fail. The problem occurs if that happens unexpectedly, since the consequence can be partial or total breakdown of a production line, destroyed equipment and even catastrophes.

Migrating towards more cost effective conditionbased and predictive maintenance (instead of sticking to the outdated concepts of reactive and periodic maintenance) has become a way to raise the overall process performance and cost efficiency. To accomplish this goal, systems for on-line and non-destructive condition monitoring (CM) have to be employed to timely alert about the onset and location of fault in the early stage [1]. Indeed, the degradation of an asset usually goes through a distinct incipient phase with some noticeable indicators, which provide advanced warning about onset of failure. However, what the operators and maintenance people indeed want to know is when to stop the machine and take accommodation actions. Reliable estimate of the remaining useful life (RUL) becomes indispensable.

In spite of significant advances in condition monitoring in the last decade in terms of methodology and key enabling technologies, yet no massive use in industrial sector has been witnessed to date [2]. There are several reasons for that, including (i) (still) relatively high cost of the design and commissioning, especially when domain specific solutions have to be adopted and (ii) the fact that traditional approaches require additional instrumentation (e.g. for rotational speed) to be implemented hence rising the cost.

Compared to CM, predicting RUL is by far more difficult problem. Only limited success has been achieved in special cases like in aeronautics and defence systems. The problem is notoriously demanding for several reasons: (i) data about overall useful life from similar items of equipment are seldom available, (ii) knowledge about degradation, i.e. wear mechanisms is incomplete and (iii) comprehensive knowledge of operating history, disturbances and past maintenance actions is usually unavailable.

The objective of the design approach presented below is to comply with the three main requirements: (i) to come up with signatures sufficiently robust to variations in the operating conditions; (ii) to set up the alarm threshold the required prior knowledge should be minimal (meaning that all the required information should 
be extracted from data in fault-free operation) and (iii) to perform condition monitoring (CM) using minimal number of sensors thus making the method both broadly applicable and financially viable.

In this paper we propose an approach to the RUL prognosis based solely on vibrational records. The idea is to exploit the relationship between the degradation phenomena in the material, the remaining life and characteristic information patterns in measured signals. The latter are obtained by statistical signal processing of signals from vibrational sensors in a way to accomplish monotonous dependance with the level of machine degradation. Evaluation of the vibrational features is based on statistical analysis of the envelope of the generated vibration [3]. State of health of the machine is determined from change in the vibrational signature by calculating the "distance" between initial and current signatures. That is achieved by evaluating the generalised Jensen-Rényi divergence of the vibrational features. Since the degradation is stochastic process, we will exploit hidden Markov models to describe the degradation phenomena. The states of the models are updated on-line and then used to simulate propagation of the future degradation and hence evaluate the probability density function of the remaining useful life.

The concept of RUL estimation above is applied to a shot blasting machine.

The rest of the paper is organised as follows. Section 2 introduces the problem related to the degradation of the machines during operation. Simple process model for RUL prediction, complemented with the health index, is presented in Section 3. Experimental results are highlighted in Section 4. The paper ends up with concluding remarks.

\section{Shot Blasting Machine}

Shot blasting machines are widely used in the process of surface cleaning where contaminants from the surface of castings are removed in order to prepare the metal parts for further finishing like, for example, painting, coating or mechanical treatment.

In shot blasting machines (Figure 1) small shots of abrasive material are fed to the turbine blades where the shots form a stream flowing along the blade length. Depending on the actual arrangement of the separating rotor and the sleeve, the flowing stream will be roughly uniform on the blades' width and length. As soon as the stream of shots leaves the blades, its direction is controlled by setting the wheel, whilst its shape changes both in width and length, thus forming a range of shot flow that hits the surface of object under treatment.
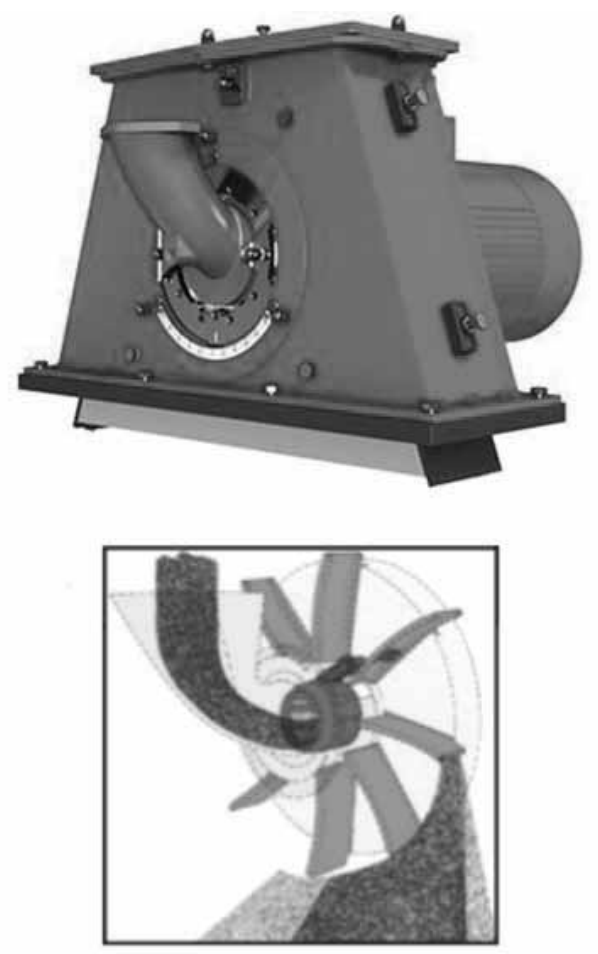

Figure 1: The shot blasting machine and illustration of the principle of operation.

The problem addressed in this paper concerns abrasive wear of the rotor blades. Abrasive grains transverse the blade from center to the periphery and their kinetic energy increases due to centrifugal forces of the rotating blade. Hence the abrasive grains scrap the surface of the blade thus forming "micro-chips", i.e. small pieces of material removed from the blade surface. With increasing number of the operating cycles the wear increases, gradually leading to the damaged blade, which can eventually break and cause downtime.

The outlook of a new blade at the beginning of the process and near failure is given in Figure 2. The problem is that it is not possible to accurately judge the level of wear on the basis of the number of cycles. Therefore it is of utmost interest for the operators to have an indicator on the level of wear in non-intrusive manner, i.e. without interrupting the blasting process.

Inference on the level of damage is done on the basis of signal analysis from vibrational sensor mounted on the housing of the machine close to the rotor bearing. 


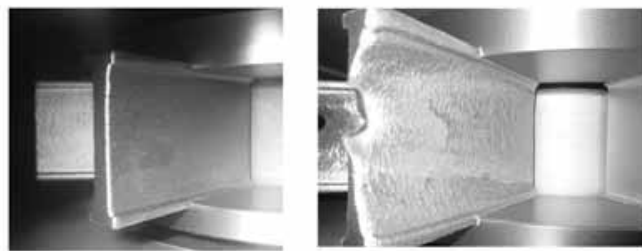

Figure 2: Turbine blade at the beginning of the operation (left) and at the end of the useful life (right).

\section{Vibrational features and health index}

\subsection{Feature extraction from vibrational signal}

Faults in the rotational machines affect the inner patterns of vibrational signals referred to as features [3]. By tracking the way these features evolve over time, it is possible to perform sufficiently accurate RUL prediction.

Wear in a turbine blade of the machine gradually results in increased imbalance of the rotor system. Vibrations resulting thereof can be viewed as the result of excitation, caused by rotor movement, on the machine eigen-structure. The resulting spectrum contains characteristic components at the frequencies $m \cdot n_{\text {blades }} \cdot f_{\text {rot }}$ where $m \in 1,2, \ldots, n_{\text {blades }}$ is the number of blades and $f_{\text {rot }}$ is rotational speed. By applying the narrow-band filtering around the characteristic frequency we get a narrow-band stochastic signal whose energy (or envelope) is Rice distributed.

Sampling of vibrational signal is performed at high frequency during short measurement sessions with an interval of 2 hours between two consecutive sessions. Changes in the probability distribution function (pdf) are characterised by calculating the "distance" between the current pdf and the reference one obtained when the machine is in nominal (healthy) state. Among several possible metrics that can be used to describe this distance, we suggest the so-called $f$-divergence measures, more precisely the generalised Jensen-Rényi (JR) divergence [4]. The rationale is simple. Instead of comparing two distributions, we compare two ensembles of distributions, one from fault-free reference condition and the other from current condition. The strength of this approach lies in the fact that comparing only two distributions is subjected to considerable fluctuations, which make final decision making difficult.

\subsection{Jensen-Rényi divergence}

The generalised Jensen-Rényi divergence (JRD), denoted by $J R_{\alpha}^{w}$ serves to quantify the dissimilarity among $n$ pdfs $\mathscr{P}_{1}, \ldots, \mathscr{P}_{n}$. It reads:

$$
J R_{\alpha}^{w}\left(\mathscr{P}_{1}, \ldots, \mathscr{P}_{n}\right)=H_{\alpha}\left(\sum_{i=1}^{n} w_{i} \mathscr{P}_{i}\right)-\sum_{i=1}^{n} w_{i} H_{\alpha}\left(\mathscr{P}_{i}\right)
$$

where $\sum_{i=1}^{n} w_{i}=1$ and $H_{\alpha}$ is the Rényi entropy:

$$
H_{\alpha}(\mathscr{P})=\frac{1}{1-\alpha} \ln \sum_{x \in \mathscr{D}} p^{\alpha}(x)
$$

with $\alpha \in[0,1]$.

The selection of weights $w_{i}$ in (1) is in principle arbitrary. If $w_{i}$ are selected uniformly i.e. $w_{i}=1 / n$, the divergence reaches maximal value [5]. JR divergence quantifies shared information among $n$ random variables. If they are identical, i.e. $\mathscr{P}_{1}=\mathscr{P}_{2}=\ldots=\mathscr{P}_{n}$, the divergence is zero.

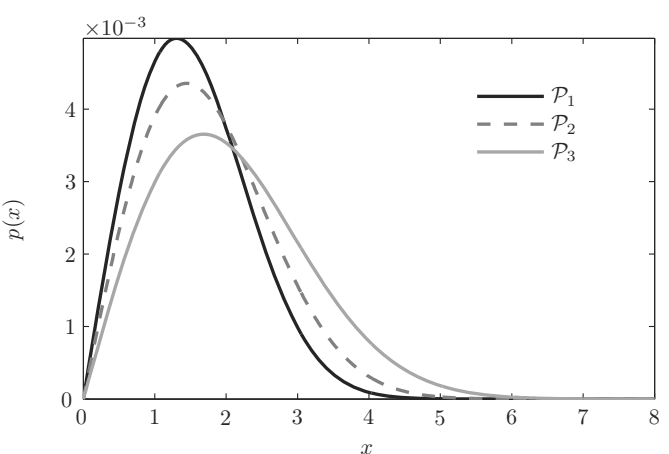

(a)

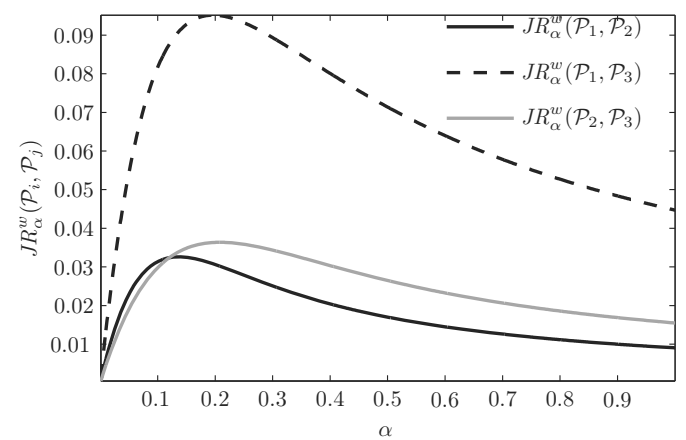

(b)

Figure 3: (a) Pdfs three random signals, and (b) pairwise JR divergence as a function of $\alpha$.

The usability of the JR divergence concept can be described with a simple example. Figure 3(a) shows 
three pdfs of Rician family. By considering the pairwise JR divergence with uniform weights, the relation (1) becomes:

$$
\begin{aligned}
J R_{\alpha}^{w}\left(\mathscr{P}_{1}, \mathscr{P}_{2}\right) & =H_{\alpha}\left(\frac{1}{2}\left(\mathscr{P}_{1}+\mathscr{P}_{2}\right)\right) \\
& -\frac{1}{2}\left(H_{\alpha}\left(\mathscr{P}_{1}\right)+H_{\alpha}\left(\mathscr{P}_{2}\right)\right),
\end{aligned}
$$

where $\mathscr{P}_{1}$ and $\mathscr{P}_{2}$ are pdfs of interest. As shown in Figure 3(b), the JR divergence corresponds to the dissimilarity between corresponding pdfs.

Figure 3(b) additionally shows the effect of the values of the parameter $\alpha$. Low value of $\alpha \approx 0$ emphasizes dissimilarity among pdfs in the lower part of the range of random variable (approximately $x \in(6,8)$ ) where pdfs do not differ much, hence low divergence values. In the middle region $(x \approx 4, \alpha \approx 0.2)$ the pdfs differ the most, hence the highest values of JR divergence. Finally, $\alpha \in(0.6,1)$ captures the region of the bulk probability masses and the divergence drops in a relatively linear manner.

\subsection{The role of weights $w_{i}$}

To allow tracking the changes in pdfs, the exponential weights $w_{i}$ are suggested in this paper. The weights $w_{i}$ are calculated using the exponential function of the following form:

$$
w_{i}=C \cdot e^{-\frac{\lambda}{n} i}
$$

where $\lambda$ is sensitivity parameter, $n$ is the number of pdfs (1), $i=1,2, \ldots, n$ and $C$ is normalising constant. One can easily see that (4) reduces to the uniform weighting for $\lambda \rightarrow 0$ and $n \rightarrow \infty$.

The influence of weights $w_{i}$ on JR divergence can be illustrated by a simple simulated example. The simulation consists of 21 Gaussian pdfs with one heaving significantly different $\mu$ as shown in Figure 4(a). The JR divergence is calculated as: $J R_{\alpha}^{w}\left(\mathscr{P}_{1}, \mathscr{P}_{2}, \ldots, \mathscr{P}_{i}\right)$, $i=1 \ldots 21$.

The rate of change in JR divergence is conditioned with the selection of weights as shown in Figure $4 \mathrm{~b}$. The most notable increase is observed if uniform weighting is applied, i.e. $w_{i}=1 / n$ [5], while exponential weighting delays the impact.

\subsection{Health index}

The concept of health index is widely used in system condition monitoring and serves to describe the aggre-
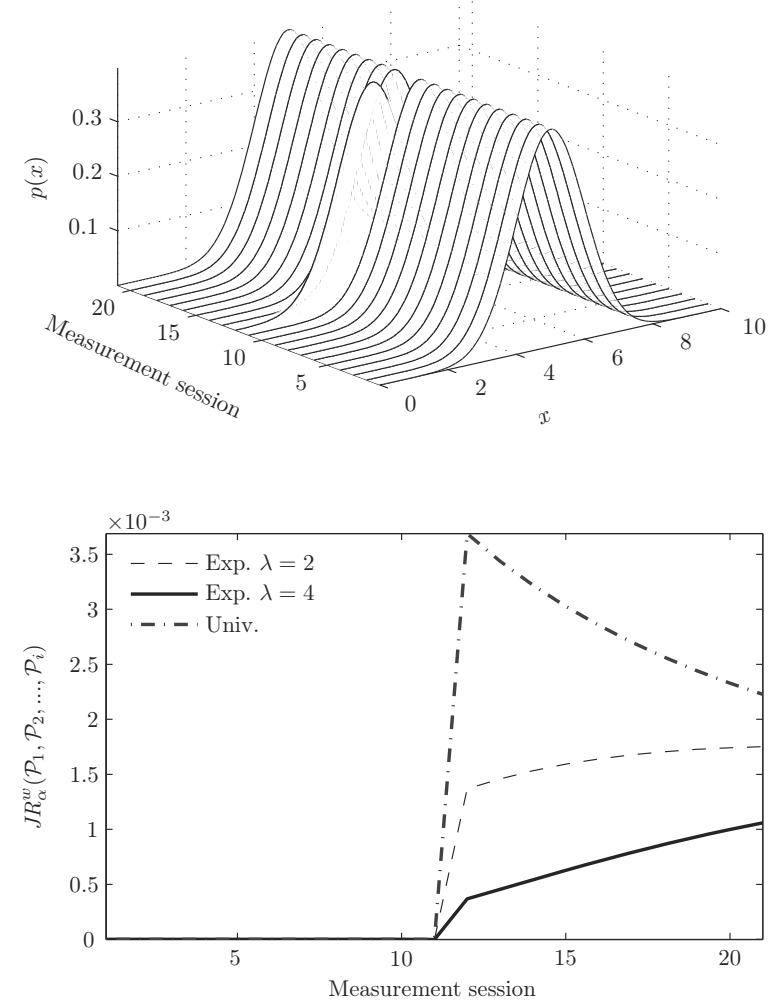

(b)

Figure 4: The evolution of JR divergence after measurement sessions. Note that all pdfs are equal except the pdf \#11. (a) Pdf's of the simulated signals associated to the measurement sessions $1, \ldots, 21$ (b) JR divergence. Up to $i<11$ there is no dissimilarity in the distribution, hence $J R_{\alpha}^{w}\left(\mathscr{P}_{1}, \mathscr{P}_{2}, \ldots, \mathscr{P}_{i}\right)=0$.

gated level of health either of a component or machine as a whole. In the case of shot blasting machines the health $H$ is perfect when the machine is new, hence $H=0$. With evolving abrasive processes on the blades, more and more surface material is removed, which results in increased vibrations. The Jensen-Rényi divergence is viewed as an appropriate metric that reflects the change in vibrational pattern caused by the level of wear in turbine blades. To find the relationship, lifelong experiments have been run in which machine operation was periodically interrupted by operators who performed invasive measurement of the blades volume. All the time during operation, the vibrations were regularly measured. The most important result of the experiment is the finding that between JRD and the extent of damage (equivalent to removed volume of blade material) 
there exists a monotone relationship. This is indicated in Figure 5. Consequently, one can adopt the health index to be equal to the normalised JRD, i.e. $H_{k}=\frac{J R D_{k}}{J R D^{*}}$ where $J R D^{*}$ stands for JRD when the machine turbine is considered worn out.

Note that health index $H$ does not rise monotonically all the time, but in the period approximately $[30,100]$ it slightly decreases. Such a behaviour looks illogical given the fact that the machine should get more and more worn with new operating cycles. The explanation lies in the fact that at the begining of the operation, the machine is not perfectly balanced. If we take into account that abrasive processes are not the same on all the blades, then asymmetry in abrasion slightly corrects the position of the center of gravity, hence resulting in lower vibrations and apparently improved condition. Such a situation changes as soon as abrasion progresses. Then asymmetrical wear in the blades results in increased imbalance and consequently raised vibrations.

\section{Stochastic Model of Abrasive Wear}

\subsection{Abrasive wear}

The key mechanism of deterioration of condition of the turbine blades is abrasive wear [6]. Each time a shot particle enters the turbine, it travels along the blade's length. Along that path it removes a small layer of the blade material of volume $\delta V$ according to the Archard's law

$$
\delta V=k \cdot \delta A \cdot \delta L,
$$

where $k$ is the wear coefficient, $\delta A$ is the contact area and $\delta L$ is the length of the path traversed by the shot particle on blade's surface.

In the ideal case, when all the blades were identical, the mass removed from each blade would be the same. Thus the center of gravity would stay at the rotational axis, which means negligible vibrations. However, due to irregularities in the particle size, angle of entry and variations of the blade's microstructure, there are minute variations in the mass removed from each blade. As a result, the generated vibrations tend to include amplitude modulations that depend on the number of blades and the rotational speed. Therefore, the intensity of these sidebands can be directly correlated with the removed volume of the blade material due to abrasive wear. Since there is no other source of vibra- tions, one can safely assume that any particular change in the vibration's signature in the lower frequency band $(<2 \mathrm{kHz})$ is due to mass loss and is therefore directly related to the blades' condition.

\subsection{Hidden Markov model}

The Archard's law (5) describes mass loss due to the blade interaction with single shot particle. During normal operation a number of particles travel along the blade's surface. During the interval of time $\left[t_{k-1}, t_{k}\right]$ the loss of volume can be written as:

$$
\Delta V_{k}=k \cdot \tilde{A}_{k} \cdot \tilde{L}_{k},
$$

where $\tilde{A}_{k}$ is the cumulative contact area of the shot particles and $\tilde{L}_{k}$ is the cumulative traversed distance. These two quantities are results of stochastic processes and, consequently, also $\Delta V_{k}$ is stochastic process. Therefore, the total volume loss at $k+1$ would be:

$$
V_{k+1}=V_{k}+\Delta V_{k}
$$

Due to surface changes, the contact area and the traversed length are expected to change over time. Therefore, based on (7), we can assume that the volume loss $\Delta V_{k}$ is a process defined by the stochastic variable defined on the set of non-negative real numbers. To consistently model such a process, several options are at disposal as for example, gamma or Weibull distribution. The problem is that in such a case recursive updates can be done only by numerical techniques. A way around is to assume that the increments $\Delta V_{k}$ fluctuate around some mean value $\mu$. The size of fluctuation can be described by a normally distributed white noise $w_{\mu} \sim \mathscr{N}\left(0, \sigma_{\mu}^{2}\right)$ such that $\sigma_{\mu} \ll \mu$. From here it follows that

$$
\Delta V_{k}-\Delta V_{k-1}=w_{\mu, k}-w_{\mu, k-1}
$$

and consequently one can write

$$
\Delta V_{k}=\Delta V_{k-1}+w_{\Delta V, k}
$$

where $w_{\Delta V, k} \sim \mathscr{N}\left(0,2 \sigma_{\mu}^{2}\right)$

Hence we get a state-space model with states $V_{k}$ and $\Delta V_{k}$. The problem now is that none of the states is available through on-line sensor reading. This can be sorted out by replacing the volume $V_{k}$ by health index $H_{k}$, which is calculated on-line from acquired vibrational records. 


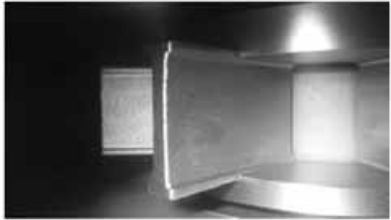

(a) Initial condition

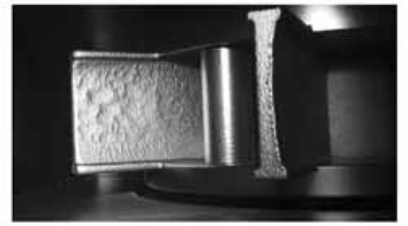

(b) Mid of experiment

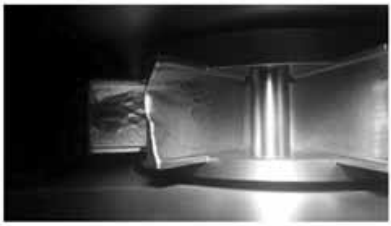

(c) End of experiment

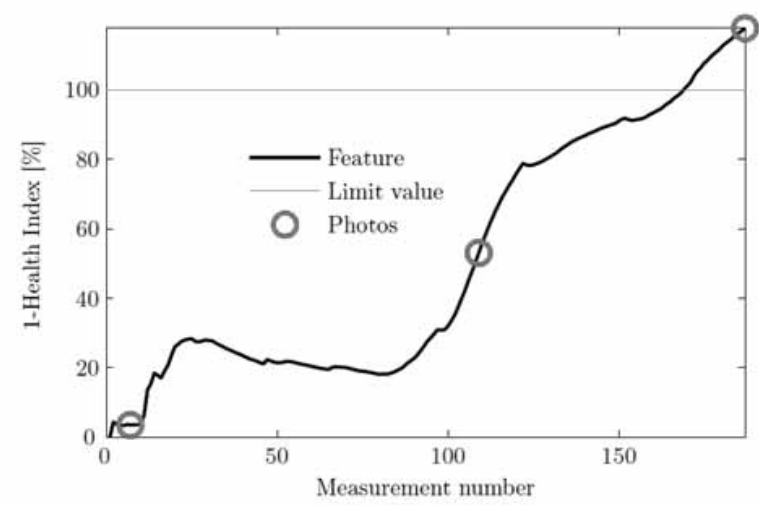

Figure 5: The relation between fault progression and evolution of health index (JR divergence): (a) at the beginning of the experiment, (b) in the middle of the experiment and (c) at the end of the experiment.

Hence the resulting state space model reads as follows

$$
\underbrace{\left[\begin{array}{c}
V_{k+1} \\
\Delta V_{k+1}
\end{array}\right]}_{\boldsymbol{x}_{k+1}}=\underbrace{\left[\begin{array}{cc}
1 & 1 \\
0 & 1
\end{array}\right]}_{\mathbf{A}} \underbrace{\left[\begin{array}{c}
V_{k} \\
\Delta V_{k}
\end{array}\right]}_{\boldsymbol{x}_{k}}+\underbrace{\left[\begin{array}{c}
0 \\
w_{\Delta V, k}
\end{array}\right]}_{\boldsymbol{w}_{k}}
$$

The measurement equation that relates system states and computable health index $H_{k}$ reads

$$
H_{k}=\underbrace{\left[\begin{array}{ll}
1 & 0
\end{array}\right]}_{\boldsymbol{C}}\left[\begin{array}{c}
V(k) \\
\Delta V_{k}
\end{array}\right]+n_{k}
$$

where $n_{k} \sim \mathscr{N}\left(0, \sigma_{n}^{2}\right)$ is white noise uncorrelated with $\boldsymbol{w}_{k}$

\subsection{Kalman fiter}

The states of the discrete model (8) can be effectively estimated using the Kalman filter approach [7,8]. The unknown states are updated at each measurement session resulting in the moments of the posterior distribution of system states $\boldsymbol{x}_{k} \sim \mathscr{N}\left(\hat{\boldsymbol{x}}_{k \mid k}, \boldsymbol{P}_{k \mid k}\right)$ as follows

1. Initialisation step: set the estimates $\hat{\boldsymbol{x}}_{0 \mid 0}=$ $\overline{\boldsymbol{x}}_{0}, \boldsymbol{P}_{0 \mid 0}, \boldsymbol{Q}=\boldsymbol{w} \boldsymbol{w}^{T}, \boldsymbol{R}=\boldsymbol{\sigma}_{n}^{2}$ from data obtained through life-long experiments on similar machines.

\section{Prediction step}

$$
\begin{aligned}
& \hat{\boldsymbol{x}}_{k \mid k-1}=\boldsymbol{A} \hat{\boldsymbol{x}}_{k \mid k-1} \\
& \boldsymbol{P}_{k \mid k-1}=\boldsymbol{A} \boldsymbol{P}_{k-1 \mid k-1} \boldsymbol{A}^{T}+\boldsymbol{Q}
\end{aligned}
$$

3. Update step: calculate the system output vector $y$ based on calculated JRD and then update the moments of state probability distribution function

$$
\begin{aligned}
\boldsymbol{K}_{k} & =\boldsymbol{P}_{k \mid k-1} \boldsymbol{C}^{T}\left(\boldsymbol{C P}_{k \mid k-1} \boldsymbol{C}^{T}+\boldsymbol{R}\right)^{-1} \\
\hat{\boldsymbol{x}}_{k \mid k} & =\hat{\boldsymbol{x}}_{k \mid k-1}+\boldsymbol{K}_{k}\left(\boldsymbol{y}_{k}-\boldsymbol{C} \hat{\boldsymbol{x}}_{k \mid k-1}\right) \\
\boldsymbol{P}_{k \mid k} & =\left(\boldsymbol{I}-\boldsymbol{K}_{k} \boldsymbol{C}\right) \boldsymbol{P}_{k \mid k-1}
\end{aligned}
$$

4. When the next measurement session appears set $k=k+1$ and go to step 2 .

\subsection{RUL predictor}

Having an updated model at a given measurement session $k$ one can simulate the possible future trajectories of the state space model (8) by Monte Carlo approach. Using realisations of random processes of noise terms $w_{\Delta, k+s}, n_{k+s}, s>0$ is is possible to calculate the corresponding trajectories of the state vector $\boldsymbol{x}_{k+s}$ and the predicted health index $H_{k+s}$. Based on that one can eas- 
ily calculate the distribution of first passage time, i.e. the time $s^{*}$ at which the health index $H$ crosses the upper bound $H^{*}$.

\section{Results of experiments}

The RUL estimation algorithm was evaluated on a shot blasting turbine in real operating environment. The blades were subjected to 400 operational hours spread over a period of 4.5 months. Vibration signals were acquired during 10 seconds long measurement sessions every two hours while the machine was in full operation. In that period, three visual inspections were performed after 10 hours of operation, at the $120^{\text {th }}$ hour and at the end of the experiment. Vibrations were measured on the bearing housing nearest to the turbine with sampling frequency of $10 \mathrm{kHz}$.

\subsection{Evolution of the health index}

The health index was calculated as JR divergence according to (1) with unifirm weights $w_{i}$. First 20 hours of operation were used as a reference point. The evolution of the health index is shown in Figure 5.

As shown in Figure 5, in the initial phase, the health index values were near zero. This is an indication that the energy distribution of the newly observed vibration is very similar to the initial 'fault-free' distribution, hence the minimal JR divergence.

The first significant increase of the JR divergence occurred around the $30^{\text {th }}$ hour of operation. After the initial increase the JR divergence gradually decreased. As said, this effect can be attributed to the runin phase of the turbine blades.

The onset of fault is visible at the $80^{\text {th }}$ hour of operation. At this point the degradation of the blade condition commenced. This is clearly indicated by the increase in the calculated JR divergence. The observed degradation was confirmed by the visual inspection performed at $120^{\text {th }}$ hour, as shown in Figure 5. The degradation trend is kept almost constant until the last fifth of the run i.e., around the $130^{\text {th }}$ hour. The calculated health index surpassed the threshold at the $180^{\text {th }}$ hour. The operation was halted at the $190^{\text {th }}$ hour with the blade condition corresponding to the estimated health index, as shown in Figure 5.

\subsection{RUL prediction}

The evolution of the calculated health index is evaluated according to the Archard's law, as described in Section 4.1. Based on results of Kalman filtering, the trajectories of future states, and consequently health index, are calculated from a set of noise realisations.

The RUL prediction based on the first 100 measurements is shown in Figure 6. At each time moment, the Kalman filter provides estimates of the posterior probability distribution of the state vector $\boldsymbol{x}_{k+s}$ and the output $y_{k+s}$. To come to the distribution of the actual RUL we perform Monte Carlo simulations of the output trajectoris. The distribution of the RUL can be evaluated from the histogram of first passage times for each simulation run. As shown in Figure 6, the proposed unscented Kalman filter (UKF) provides left skewed RUL estimates. The $3 \sigma$ confidence interval is sufficiently narrow and corresponds to the actual evolution of the health index.

For proper assessment of the model's accuracy, the RUL estimates should be plotted versus a theoretically expected RUL. Typically, the theoretical RUL is expected to be a linear function with gradient -1 . This is shown in Figure 7. Note that during the first 2/3 of the operational life the RUL prediction is not reliable. However, in the last third of the life, predictions become rather accurate meaning that roughly 2 months before the blades are fully worn the operators have reliable information, which could be used to plan the maintenance actions at a convenient occasion in a way that do not disturb regular production (for example, during a weekend or night shift).

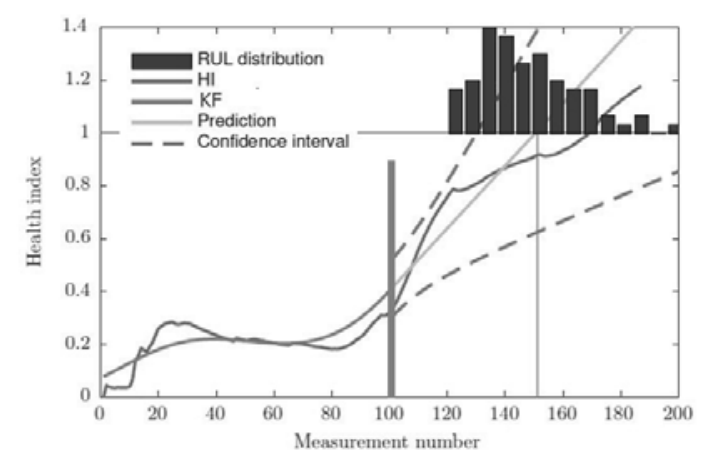

Figure 6: RUL prediction at the $100^{\text {th }}$ measurement 


\section{Conclusion}

The proposed feature based on JR divergence is shown to be sufficiently sensitive to perform accurate condition monitoring of shot blasting machines. Furthermore, it is shown that the evolution of the JR divergence can be directly related to the removed mass from the turbine's blades due to abrasive wear. As a result, the evolution profile can be described through Archard's law of abrasive wear. Based on this result, accurate RUL prediction is be achieved by estimating the models's states using computationally simple Kalman filter and Monte Carlo simulations over noise realisations.

\section{Acknowledgements}

The authors acknowledge the support of the Slovenian Research Agency through the Research Programme P2-0001 and the Research Project L2-7663.

\section{References}

[1] Lee J, Wu F, Zhao W, Ghaffari M, Liao L, Siegel D. Prognostics and health management design for rotary machinery systems-reviews, methodology and applications. Mechanical Systems and Signal Processing, vol. 42, no. 1-2, pp. $314-334,2014$.

[2] Jablonski A. Condition monitoring systems readressed and revised. vol. Nov., p. 2, 2015.

[3] Boškoski P, Juricic D. Fault detection of mechanical drives under variable operating conditions based on wavelet packet rényi entropy signatures. Mechanical Systems and Signal Processing, vol. 31, pp. 369-381, 2012.

[4] Basseville M. Divergence measures for statistical data processing - an annotated bibliography. Sig-nal Processing, vol. 93, pp. 621-633, 2013.

[5] Hamza A, Krim H. Image registration and segmentation by maximizing the jensen-rényi divergence. In Energy Minimization Methods in Computer Vision and Pattern Recognition (A. Rangarajan, M. Figueiredo, and J. Zerubia, eds.), vol. 2683 of Lecture Notes in Computer Science, pp. 147-163, Springer Berlin Heidelberg, 2003.

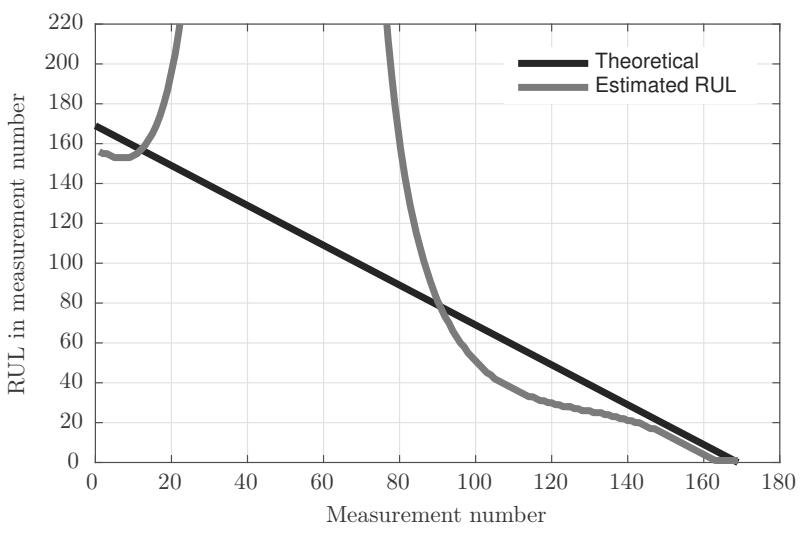

Figure 7: Performance of the RUL prediction algorithm.

[6] Bisson EE. Adhesive, abrasive, corrosive, and surface fatigue wear modes. Tech. Rep. TM X52426, NASA Lewis Research Center, Cleveland, OH, United States, 1968.

[7] Verhaegen M, Verdult V. Filtering and System Identification. Cambridge University Press, Cambridge, UK, 2007.

[8] M. Gašperin M, Juricic D. Application of unscented transformation in nonlinear system identification," \{IFAC\} Proceedings Volumes, vol. 44, no. 1, pp. 4428 - 4433, 2011. 18th \{IFAC \} World Congress. 